\title{
Diluting 5-fluorouracil with normal saline reduces patient discomfort
}

\begin{abstract}
Keith Ong ${ }^{1,2,3,4}$, Ru Min Ong ${ }^{5}$, Daisy Shu ${ }^{6,7}$
'Department of Ophthalmology, Northern Clinical School, University of Sydney, Sydney, NSW, Australia; '2 Department of Ophthalmology, Royal North Shore Hospital, Sydney, NSW, Australia; ${ }^{3}$ Department of Ophthalmology, Sydney Adventist Hospital, Sydney, Australia; ${ }^{4}$ Department of Ophthalmology, Chatswood Private Hospital, Sydney Australia; ${ }^{5}$ Medical Faculty, University of NSW, Sydney, Australia; ${ }^{6}$ Discipline of Anatomy \& Histology, Bosch Institute, University of Sydney, NSW, Australia; ' Save Sight Institute, University of Sydney, NSW, Australia
\end{abstract}

5-Fluorouracil (5-FU) is an antimetabolite and is used to improve the success rate of trabeculectomies by inhibition of subconjunctival fibrosis. It can be administered postoperatively as a subconjunctival injection or given intraoperatively via cellulose sponge soaked in 5-FU and placed between the sclera and conjunctival flap. The advantage of injecting 5-FU subconjunctivally postoperatively is that the dose and frequency can be titrated according to patients' needs. Subconjunctival injection may be uncomfortable and this could be due to the alkaline nature of 5-FU (pH 9.20) and its osmolarity of $384 \mathrm{mmol} / \mathrm{l}$. It would be beneficial if this discomfort can be minimised as the injection may need to be given several times.

A small study was performed to evaluate whether dilution of 5-FU with normal saline can improve discomfort. The cohort consisted of 31 patients who required subconjunctival 5-FU injection after trabeculectomy and needling of filtration bleb. They were given plain $0.2 \mathrm{ml} \mathrm{5-FU}(50 \mathrm{mg} / \mathrm{ml})$ on one occasion and diluted 5 -FU $(0.2 \mathrm{ml} 5$-FU and $0.2 \mathrm{ml}$ normal saline) on another. Patients were administrated topical anaesthesia with Alcaine $0.5 \%$ (Proparacaine hydrochloride ophthalmic solution; Alcon, Fort Worth, TX, USA) and lignocaine 2\% before the injection. The duration of the pain was recorded in seconds.

The average pain duration when plain 5-FU was administered subconjunctivally was 9.77 seconds, discomfort duration ranging from no pain to maximum pain lasted for 40 seconds. With diluted 5-FU, the average pain was 1.06 seconds, with discomfort duration ranging from no pain to maximum pain of 11 seconds among different patients. The average improvement of pain with the use of diluted 5-FU compared to plain 5-FU was 8.71 seconds.

Correspondence: Dr. Keith Ong, 2 Railway Avenue, Eastwood, NSW 2122, Australia.

E-mail: keithong@optusnet.com.au 
Three patients (9.7\%) experienced no pain with both plain 5-FU and diluted 5-FU; 25 patients $(80.6 \%)$ who had pain with plain 5-FU experienced no pain when 5 -FU was diluted. Three patients (9.7\%) experienced pain with both plain 5-FU and diluted 5-FU but had less pain with the use of diluted 5-FU.

This small study shows that the pain of subconjunctival 5-FU injection is reduced when 5 -FU is diluted with normal saline. The $\mathrm{pH}$ was measured by $\mathrm{pH}$ paper and pH meter (Edge pH meter; HI2020 Hanna Instruments, Woonsocket, Rhode Island). The pH of plain 5-FU is 9.20 and that of normal saline is 8.32 . When combined together, the $\mathrm{pH}$ of diluted $5-\mathrm{FU}$ is 9.09 .

Osmolarity of plain 5-FU is $384 \mathrm{mmol} / \mathrm{l}$ and osmolarity of normal saline is 286 $\mathrm{mmol} / \mathrm{l}$. When $5-\mathrm{FU}$ is diluted $50 \%$ with normal saline, the resultant osmolarity should be $335 \mathrm{mmol} / \mathrm{l}$. This value is closer to plasma osmolality of $295 \mathrm{mOsm} / \mathrm{kg}$.

Hence, this study demonstrates that if patients complain of pain and discomfort with subconjunctival 5-FU injections, diluting 5-FU with normal saline (1:1) can reduce the discomfort.

\section{Conflict of interest}

There is no conflict of interest and no funding was received for this work. 